\title{
Essentiality and functional analysis of type I and type III pantothenate kinases of Mycobacterium tuberculosis
}

\begin{abstract}
Correspondence
Umender Sharma

umender.sharma@astrazeneca. com
\end{abstract}

Received 20 April 2010

Revised 20 June 2010

Accepted 22 June 2010

\author{
Disha Awasthy, Anisha Ambady, Jyothi Bhat, Gulebahar Sheikh, \\ Sudha Ravishankar, Venkita Subbulakshmi, Kakoli Mukherjee, \\ Vasan Sambandamurthy and Umender Sharma
}

AstraZeneca R \& D, 'Avishkar' Bellary Road, Hebbal, Bangalore, India

\section{INTRODUCTION}

Mycobacterium tuberculosis, the causative agent of tuberculosis, infects approximately one-third of the world's population and causes morbidity and mortality in a large number of people, mostly in underdeveloped countries (Hoft, 2008). Though it is possible to control the disease with a combination of drugs, the long duration of treatment often leads to non-compliance resulting in relapse of active disease and emergence of drug-resistant M. tuberculosis. The presence of drug-resistant bacteria in the population poses a serious threat to mankind as a significant number of these bacteria are resistant to multiple drugs, leaving physicians with little choice for prescribing drugs (Chan \& Iseman, 2008). Hence there is an urgent need to discover new drugs and vaccines for controlling tuberculosis. As a result of development of

Abbreviations: ara, arabinose; BMDM, bone-marrow-derived macrophages; CoA, coenzyme A; DCO, double crossover; HRP, horseradish peroxidase; IFN- $\gamma$, gamma-interferon; KO, knock-out; PBST, PBS containing $0.1 \%$ Tween-20; SCO, single crossover; ts, temperature sensitive. good genetic tools and the availability of the complete genome sequence of $M$. tuberculosis (Cole et al., 1998), there have been rapid advances in understanding the molecular mechanisms of pathogenesis of M. tuberculosis. A number of new inhibitors of M. tuberculosis have also been reported which are undergoing pre-clinical or clinical evaluations, but are yet to be approved for regular use in patients (Rivers \& Mancera, 2008). Efforts to design novel vaccines have met with limited success (Hoft, 2008). In order to sustain the efforts towards tuberculosis control there is a need to identify and validate novel M. tuberculosis proteins as drug or vaccine candidates, which will help in designing novel therapeutic approaches for controlling this important disease.

Since coenzyme A (CoA) is an essential acyl group carrier co-factor required for growth of various organisms, the enzymes of the CoA biosynthesis pathway have been thought to be good targets for designing inhibitors against bacteria, parasites and fungi (Gerdes et al., 2002; Spry et al., 2008). Pantothenate kinases catalyse the first step in the biosynthesis of CoA. In Escherichia coli, pantothenate 
kinase activity has been proposed to be the regulatory step in biosynthesis of CoA (Jackowski \& Rock, 1981; Rock et al., 2003), thus making it an attractive target for designing inhibitors. There are three isoforms of pantothenate kinase known in various organisms. The majority of the bacteria possess type I enzyme (e.g. E. coli PanK or EcCoaA) while eukaryotes (e.g. murine enzyme, MmPanK) and a few bacteria (e.g. Staphylococcus aureus SaCoaA) carry a type II enzyme (type II PanK). Although type I and type II isoforms do not possess a high degree of amino acid homology, they both share common regulatory mechanisms and are inhibited by $\mathrm{CoA}$ and its thioesters. However, in this aspect, the SaCoaA is an exception, as its activity is not regulated by CoA (Leonardi et al., 2005). The type III isoform discovered recently (CoaX), which lacks any amino acid similarity to type I and type II isoforms has been identified in a number of bacteria including Bacillus subtilis, Helicobacter pylori, Pseudomonas aeruginosa and Bordetella pertussis. Enzymic characterization of CoaX from B. subtilis and H. pylori demonstrated that the type III isoform exhibits distinct biochemical properties such as lack of feedback regulation by CoA and an inability to accept pantothenic acid metabolites (pantothenamides) as substrates (Brand \& Strauss, 2005). Though there are no known anti-bacterials which inhibit the CoA biosynthesis pathway, pantothenate analogues and amide derivatives, pantothenol, $\mathrm{N}$-pentylpantothenamide and $\mathrm{N}$-heptylpantothenamide possess antibiotic activity against $E$. coli, demonstrating the essential nature of this pathway (Snell \& Shive, 1945; Choudhry et al., 2003; Zhang et al., 2004). A number of pantothenic acid analogues have been shown to possess anti-malarial activity (Saliba et al., 2005; Spry et al., 2005). In addition, specific inhibitors of another enzyme in the CoA biosynthesis pathway of $E$. coli, CoaD (PPAT, phosphopantotheine adenyltransferase) have also been reported (Zhao et al., 2003). Based on these observations, it is fair to speculate that inhibition of pantothenate kinase activity in $M$. tuberculosis will lead to inhibition of cell growth.

The coaA gene has been found to be essential by gene inactivation studies in a number of bacteria including E. coli and Salmonella typhimurium (Dunn \& Snell 1979; Vallari \& Rock, 1987). In S. aureus, which possesses a type II enzyme, pantothenamides were shown to inhibit the growth of the bacteria, thereby demonstrating the essential nature of this enzyme (Choudhry et al., 2003). In B. subtilis, the coaA gene could be inactivated and it was discovered that another gene, designated coaX, could complement the loss of coaA (Yocum \& Patterson, 2004). However, double knock-out of coaA and coaX was found to be lethal to the cell, demonstrating that at least one isoform of pantothenate kinase has to be functional. The ability of either of these pantothenate kinases to support the growth of B. subtilis points towards redundancy of this function in this species of bacteria. However, in B. anthracis, the coaX gene has been found to be essential even in the presence of a gene encoding a type II pantothenate kinase, indicating that their functions are not interchangeable in this organism. (Paige et al., 2008). A homologue of CoaX in Bordetella pertussis, BvgS has been shown to be essential for viability (Wood \& Friedman, 2000). Thus, from genetic and biochemical studies on various bacteria, it seems that all three types of pantothenate kinases are independently capable of performing the essential function of phosphorylating pantothenate and any one of them could be essential for viability. The reason for the presence of single or multiple isoforms of pantothenate kinases in different bacteria is not clear. The situation in M. tuberculosis is similar to B. subtilis, wherein, in addition to coaA, the coaX gene is also present. In order to identify the essential pantothenate kinase in $M$. tuberculosis for the purpose of utilizing it as a drug target, we have attempted to inactivate coaA and coaX genes individually. Surprisingly, coaX could be inactivated in $M$. tuberculosis without any deleterious effects under in vitro or in vivo growth conditions, proving it to be unattractive for drug discovery. CoaA, on the other hand, was found to be essential and hence could be a valid drug target in M. tuberculosis.

\section{METHODS}

Bacterial strains. The strains used in this study are listed in Table 1.

M. tuberculosis culture conditions. M. tuberculosis H37Rv (ATCC 27294 ) cultures were routinely grown in $7 \mathrm{H} 9$ or $7 \mathrm{H} 11$ medium (Difco) containing ADC enrichment. Hygromycin $\left(50 \mu \mathrm{g} \mathrm{ml}^{-1}\right)$ or kanamycin $\left(20 \mu \mathrm{g} \mathrm{ml}^{-1}\right)$ was added to the culture medium when required.

DNA amplification by PCR. The single crossover (SCO) and double crossover (DCO) recombinants in M. tuberculosis were screened by PCR using Taq DNA polymerase. Single isolated bacterial colonies resuspended in $50 \mu \mathrm{l}$ TE (10 mM Tris, $1 \mathrm{mM}$ EDTA) were boiled for $10 \mathrm{~min}$. An aliquot of the cell lysate $(5 \mu \mathrm{l})$ was added to $25 \mu \mathrm{l} \mathrm{PCR}$ mixture. Denaturation and extension reactions were performed at 94 and $72{ }^{\circ} \mathrm{C}$, respectively, for $30 \mathrm{~s}$ each. The annealing temperature and extension time for each PCR were determined by considering the melting temperature of the primer pair and length of the PCR product, respectively.

RT-PCR. $M$. tuberculosis cultures were grown in $7 \mathrm{H} 9$ medium until the $\mathrm{OD}_{600}$ reached 0.2. The cell suspensions in Trizol (Invitrogen) were subjected to bead beating using $0.1 \mathrm{~mm}$ Silica/Zirconia beads (Mini bead beater Biospec Products) at 5000 r.p.m. with $30 \mathrm{~s}$ pulses. The samples were extracted with chloroform followed by 2-propanol precipitation at $4{ }^{\circ} \mathrm{C}$ overnight. The nucleic acid pellet obtained after centrifugation was resuspended in RNase-free water and treated with DNase I (Ambion) for $30 \mathrm{~min}$ at $37^{\circ} \mathrm{C}$. The total RNA was purified using an RNeasy column (Qiagen). Purified RNA ( $1 \mu \mathrm{g}$ ) was used to synthesize cDNA by a reverse transcriptase (RT) reaction using reverse primers, $0.5 \mathrm{mM}$ dNTP mix and Superscript II (Invitrogen) at $37{ }^{\circ} \mathrm{C}$ for $60 \mathrm{~min}$ in a $30 \mu \mathrm{l}$ reaction volume. An RT-minus reaction was set up as a negative control. An aliquot of $1.5 \mu \mathrm{l}$ of the RT reaction mix was further used to set up the individual PCRs to amplify fragments of coaA and coaX as described above. The sequences of the primers used were as follows: coaA forward primer (TCoARTF) 5'-ACGCTCATGGTGTCGGATCTGTT-3', reverse primer (TCoARTR) 5'-GCAGCCGGTTAATGGAATGATC-3'; coaX forward primer (472) 5'-AGTGGCGGATACGCACCGAAT-3', reverse primer (473) 5'-GGCATCGGAAGACACCTGCAC- $3^{\prime}$. 
Table 1. Strains and plasmids used in this study

\begin{tabular}{|c|c|c|}
\hline Strain/plasmid & Characteristics & Source/Ref \\
\hline E. coli $\mathrm{DH} 5 \alpha$ & endA1 hsdR17 supE44 recA1 relA1 lacZYA-argF & Lab. stock \\
\hline E. coli DV62 & panD2 gyrA21 relA1 spoT1 metB zif-2009:: Tn10 coaA15(ts) & CGSC \\
\hline pGOAL19 & E. coli ori, sacB, $\beta$-gal, $\mathrm{Hyg}^{\mathrm{r}}$ & Parish \& Stoker (2000) \\
\hline pAZI272 & Integrating mycobacterial expression vector & Awasthy et al. (2009) \\
\hline pAZI0222 & $h y g^{r}$ cassette cloned into NruI site of pAZI0221 & This study \\
\hline pAZI0228 & BamHI-HindIII fragment from pAZI0221 cloned into similar sites of pGOAL19 & This study \\
\hline pAZI0290 & pGOAL19 with a deletion of lac $Z$ gene & This study \\
\hline pAZI0294 & $\begin{array}{l}\Delta c o a X \text { with upstream and downstream sequences cloned into BglII-NcoI sites of } \\
\text { pAZI0290 }\end{array}$ & This study \\
\hline pBAD24 & Arabinose inducible E. coli expression vector & Guzman et al. (1995) \\
\hline pAZI0371 & His:CoaX coding DNA fragment cloned into NcoI-HindIII sites of pBAD24 & This study \\
\hline pAZI0372 & His:CoaA coding DNA fragment cloned into NcoI-HindIII sites of pBAD24 & This study \\
\hline pJL28 & Mycobacterial plasmid carrying a gene coding for L5 phage excisionase & Parish et al. (2001) \\
\hline pAZI0288 & $\mathrm{Gm}^{\mathrm{r}}$ marker of pJL28 replaced by $h y g^{r}$ gene & This study \\
\hline
\end{tabular}

Plasmid constructs. The plasmids used in this study are listed in Table 1. The preparation of constructs used for gene knock-out (KO) of coaA (Rv1092c) and coaX (Rv3600c) was outsourced to Bangalore Genei (India). The strategy followed for making these constructs was as follows. The recombination substrate for creating a deletion in the coaA gene of $M$. tuberculosis consisted of $803 \mathrm{bp}$ of the region upstream of coaA, a 739 bp deletion (7-746) in coaA followed by $194 \mathrm{bp}$ of the $3^{\prime}$-end of the gene and $884 \mathrm{bp}$ of the downstream region cloned into a suicide vector, pGOAL19. A 1693 bp DNA fragment generated by overlapping PCR was cloned into the BamHIHindIII site of pGOAL19 to obtain pAZI0228. The coaX (Rv3600c) $\mathrm{KO}$ construct [consisting of $609 \mathrm{bp}$ of the upstream region, $237 \mathrm{bp}$ of the $5^{\prime}$-end of the gene, $181 \mathrm{bp}$ deletion, $401 \mathrm{bp}$ of the $3^{\prime}$-end of the gene (total gene length, $819 \mathrm{bp}$ ) and $433 \mathrm{bp}$ of downstream region] was generated similarly and cloned into the NcoI-BglII sites of pAZI0290 to obtain pAZI0294. The DNA sequences of the cloned fragments were confirmed by sequencing. For generating constructs for complementation in M. tuberculosis, the coaA and coaX genes were amplified from the M. tuberculosis genomic DNA by PCR using highfidelity Taq DNA polymerase (Phusion, Finnzyme). The primer sequences were as follows: coaA forward (328) 5'-ATAGGTCCATCGCGGCTTAGCGAGCCG-3', reverse (329) 5'-ACTGAAGCTTTTACAGCTTGCGCAGCCG-3'; coaX forward (638) 5'-ATCCAGCTGCACTGCTGGCGATTGACGTCC-3', reverse (639) 5' -CTAGTTAACTCAGCGCGCCGTCTTGAGC-3'. The amplified PCR products were cloned into BamHI-HindIII (coaA) or PvuII-HpaI (coaX) sites of pAZI272 to obtain pAZI0234 and pAZI0297, respectively. For expressing CoaX in E. coli, the coaX gene was amplified by PCR from M. tuberculosis genomic DNA and cloned into the NdeI-BamHI sites of pET15b to obtain pAZI9015. The DNA sequences of the cloned coaA and coaX genes were confirmed by sequencing (microsynth, Switzerland). For growth rescue of a temperature-sensitive (ts) mutant of E. coli at a non-permissive temperature, coaA and coaX genes were cloned into the arabinose-inducible expression vector pBAD24. For this, a $1.2 \mathrm{~kb}$ DNA fragment encompassing the coaA gene from a T7 expression construct of His::CoaA in pET15b
(pAZI9004) was cloned into the NcoI-HindIII sites of pBAD24 to obtain pAZI0372. Similarly, the coaX gene from a T7 expression construct pAZI9015 was cloned into NcoI-HindIII sites of pBAD24 to generate pAZI0371.

Gene KO and excision in M. tuberculosis. The recombinant plasmids were electroporated into $M$. tuberculosis by the procedure described by Wards \& Collins (1996). The gene KO was performed by using the two-step method as described by Parish \& Stoker (2000). For excisionase activity, pAZI0288 was transformed into the wild-type M. tuberculosis and the coaA mutant strains and plated in the presence of hygromycin. The transformation frequencies were compared, and the excision of the integrated plasmid was monitored by PCR.

Southern blotting. Genomic DNA from the wild-type or mutant $M$. tuberculosis strains was digested with KpnI (for coaA) or SphI (for coaX) and separated by electrophoresis on $0.8 \%$ agarose gels. The probe was generated by amplification of DNA fragments encompassing coaA and coaX genes by PCR, as shown in Figs 1 and 2. The primer sequences were as follows: coaX forward (471) 5' -AGCGCATGCGGCAACGGCTGG3', reverse (578) 5'-GTGGCGCGGTGGATCTTCGTC-3'; and coaA forward (422A) 5'-TCACACTTCCACCACTACGCG-3', reverse (423) 5'-ATCGTTGCCGCTGAATTGGC-3'. The probes were labelled with fluorescein by random prime labelling (Roche). DNA transfer, hybridization and washing were performed by using the protocol provided by the manufacturer (GE Healthcare). The bound DNA probes were detected by horseradish peroxidase (HRP)-conjugated antifluorescein antibodies (1:2000 dilution) using chemiluminescence.

Complementation in $\boldsymbol{E}$. coli DV62. pAZI0371, pAZI0372 and pBAD24 (vector) were transformed into E. coli DV62, plated on LB plates containing ampicillin $\left(50 \mu \mathrm{g} \mathrm{m}^{-1}\right)$ and $0.5 \mathrm{mM}$ arabinose (ara) and incubated at 30 or $42{ }^{\circ} \mathrm{C}$ for $24 \mathrm{~h}$. Alternatively, colonies from plates incubated at $30{ }^{\circ} \mathrm{C}$ were picked up and grown in broth until the $\mathrm{OD}_{600}$ reached 0.2 , and dilutions were plated on LB plates containing ampicillin and ara at 30 and $42{ }^{\circ} \mathrm{C}$ for $24 \mathrm{~h}$. 
(a)

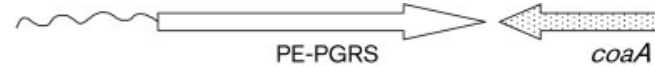

(b)

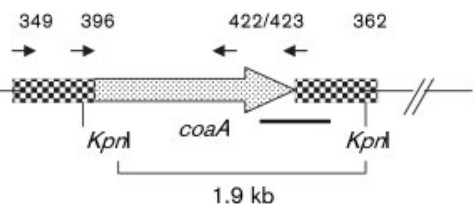

(c)

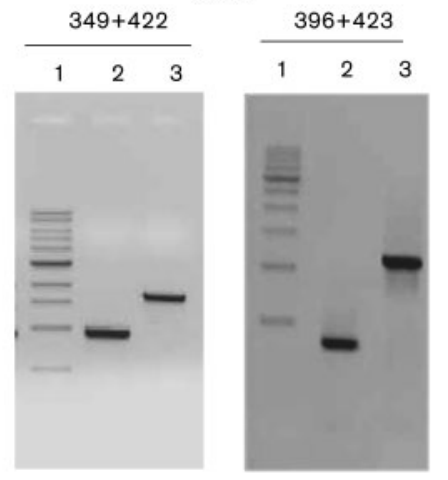

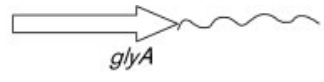

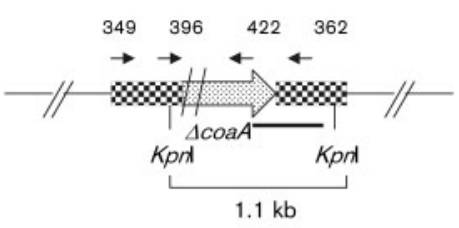

(d)

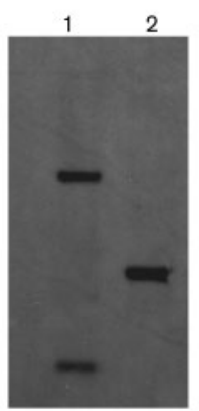

Fig. 1. Gene $\mathrm{KO}$ of $\operatorname{coa} A$. (a) Genomic organization of $\operatorname{coa} A$ in the $M$. tuberculosis genome. (b) Comparison of the sizes of wildtype and the mutant alleles of $\operatorname{coa} A$, the binding sites of primers used for amplification of DCO recombinants by PCR and the position of restriction enzymes used for digestion of DNA for Southern blotting. The probe used for hybridization is shown as solid lines. (c) Analysis of Mtb/coaA/KO by PCR. Lanes: 1, molecular size marker; 2, Mtb/coaA/KO; 3, H37Rv. (d) Southern blot of Mtb/coaA/KO (lane 1) and H37Rv (2). The primer sequences are: 349, 5'-GATGATCACCTTCGGGCGGAATTC- 3'; 396, 5'-CCCGTCCCTGCAAGACTGACA-3'; 422, 5'-GCGTAGTGGTGGAAGTGTGA-3'; 423, 5-ATCGTTGCCGCTGAATTGGC-3'; 362, 5'-CTAACGGCGTCAGCAGCACCAATG-3'.

\begin{abstract}
Western blotting. The cytosolic fractions of cell lysates were separated by $12 \%$ SDS-PAGE and transferred onto a nitrocellulose membrane. BSA (3\%) in PBS containing $0.1 \%$ Tween-20 (PBST) was used for blocking and the blot was incubated in the presence of rabbit anti-CoaX antibodies diluted 1:200000 for $1 \mathrm{~h}$. After washing with PBST, HRP-conjugated anti-rabbit antibodies (diluted 1:200 000) were added to the blot and the incubation was continued for $1 \mathrm{~h}$. The blot was washed with PBST and the induced proteins were detected by chemiluminescence using ECL Western blotting kit (GE Healthcare) by using the method provided by the manufacturer.
\end{abstract}

Purification of M. tuberculosis CoaA and CoaX. E. coli BL21 (DE3) transformed with pAZI9015 was grown in LB to $\mathrm{OD}_{600} 0.5$, and was induced with $1 \mathrm{mM}$ IPTG for $3 \mathrm{~h}$. The cell pellet obtained from 21 IPTG-induced cultures was resuspended in $50 \mathrm{ml}$ buffer A (50 mM Tris/HCl, pH 7.5, $300 \mathrm{mM} \mathrm{NaCl}, 1 \mathrm{mM} \mathrm{DTT}, 10 \%$ glycerol, $1 \mathrm{mM}$ PMSF and $100 \mu \mathrm{g}$ lysozyme $\mathrm{ml}^{-1}$ ). The resuspended cells were kept on ice for $30 \mathrm{~min}$ and were lysed by sonication (Branson sonifier-450) at $4{ }^{\circ} \mathrm{C}$. The cell lysate was centrifuged at $100000 \mathrm{~g}$ for $1 \mathrm{~h}$. The supernatant was recovered and loaded on a Ni-NTA column, pre-equilibrated in buffer $\mathrm{A}$. The flow-through was collected and the column was washed with $50-100 \mathrm{ml}$ wash buffer (buffer A containing $10 \mathrm{mM}$ imidazole). The bound proteins were eluted with elution buffer (buffer A containing $300 \mathrm{mM}$ imidazole) and dialysed against gel filtration buffer $(50 \mathrm{mM}$ Tris/ $\mathrm{HCl}, \mathrm{pH} 7.5,500 \mathrm{mM} \mathrm{NaCl}, 2 \mathrm{mM}$ DTT, $10 \%$ glycerol and $1 \mathrm{mM}$ EDTA) overnight at $4{ }^{\circ} \mathrm{C}$. The dialysed protein sample $(30 \mathrm{ml})$ was concentrated to $2 \mathrm{ml}$ and loaded on a pre-equilibrated gel filtration column (Superdex pg 200) at a flow rate of $1.0 \mathrm{ml} \mathrm{min}{ }^{-1}$. Fractions of $5 \mathrm{ml}$ were collected, and fractions E1418 were pooled and concentrated to $1 \mathrm{ml}$ using a Millipore concentrator (MWCO-10 kDa). The protein sample was stored at $70{ }^{\circ} \mathrm{C}$ in $0.25 \mathrm{ml}$ aliquots. For CoaA expression, E. coli BL21 star (DE3) transformed with pAZI9004 was grown in LB medium at $25{ }^{\circ} \mathrm{C}$ overnight in the absence of the inducer, as the optimum amount of protein was expressed under these conditions. CoaA was purified by using the procedure described above, except that $100 \mathrm{mM} \mathrm{NaCl}$ was used in the gel filtration buffer.

Biochemical assay. Pantothenate kinase activity was measured by detecting adenosine diphosphate formation using pyruvate kinase and lactate dehydrogenase as described previously (Yang et al., 2008). The activity was assayed in a $100 \mu \mathrm{l}$ reaction mixture containing $50 \mathrm{mM}$
PIPES NaOH, pH 7.0, $25 \mathrm{mM} \mathrm{KCl,} 20 \mathrm{mM} \mathrm{MgCl}_{2}, 0.1 \mathrm{mM}$ EDTA, $2 \mathrm{mM}$ DTT, $0.002 \%$ Brij-35, $0.5 \mathrm{mM}$ PEP, $0.24 \mathrm{mM}$ NADH, 10 units $\mathrm{PK} / \mathrm{LDH} \mathrm{ml} \mathrm{m}^{-1}$ (Sigma), $0.12 \mathrm{mM}$ ATP and $0.3 \mathrm{mM}$ D-pantothenate. The reaction was started by the addition of CoaA $(20-100 \mathrm{nM})$ or CoaX $(100 \mathrm{nM}-3.2 \mu \mathrm{M})$ to the reaction mix. The reaction was monitored by observing the change in $A_{340}$ for $60 \mathrm{~min}$ at $25^{\circ} \mathrm{C}$ in a Spectramax spectrophotometer. The velocity of the reaction was calculated from the linear range of the reaction by estimating the amount of NADH converted per second. For this purpose, the molar absorption coefficient of NADH was taken as $6220 \mathrm{M}^{-1} \mathrm{~cm}^{-1}$, and the path length considered for a $100 \mu \mathrm{l}$ volume was $0.275 \mathrm{~cm}$. The catalytic constant $\left(k_{\text {cat }}\right)$ was determined by using the formula $k_{\text {cat }}=V_{\text {max }} / E_{\mathrm{t}}$, where $V_{\max }$ is the maximum enzyme velocity, and $E_{\mathrm{t}}$ is the total enzyme concentration.

Growth of M. tuberculosis H37Rv and coaX KO mutant in macrophages. Intracellular infection and survival kinetics of mycobacterial strains were investigated in gamma-interferon (IFN- $\gamma$ )activated bone-marrow-derived macrophages (BMDM) in a 24-well plate format as described by Muñoz-Elías \& McKinney (2005). Briefly, bone marrow cells were flushed from mouse femurs and cultured in complete RPMI 1640 medium (Gibco Laboratories) with $20 \%$ L929 conditioned medium for 5 days. The macrophage monolayers were activated by addition of $25 \mathrm{ng}$ recombinant IFN- $\gamma \mathrm{ml}^{-1}$ (Sigma) for $48 \mathrm{~h}$. Activated and washed BMDM monolayers were used for infection. All macrophage infection studies were performed in RPMI 1640 medium (Cell Culture Technologies). Monolayers were infected with M. tuberculosis $\mathrm{H} 37 \mathrm{Rv}$ and coaX KO mutant strains individually at an m.o.i. of $1: 10$. Two hours post-infection, the monolayer was washed with PBS and replaced with RPMI 1640 medium. A set of BMDM cells infected with wild-type and coaX KO mutant were lysed on day 0, 3, 7 and 10 . The lysates were plated onto 7H11 agar plates.

Mouse infection. The Institutional Animal Ethics Committee, registered with the government of India (registration no. CPCSEA 99/5) approved all animal experimental protocols and usage. Six- to eight-week-old BALB/c mice purchased from the National Institute of Nutrition, Hyderabad, India, were randomly assigned to cages at five mice per cage, with the restriction that all cage members were within a 1-2 g weight of each other. They were allowed to acclimatize for 2 weeks before intake into experiments. For intravenous infection, frozen bacterial samples were thawed, centrifuged and washed in PBS, 
(a)
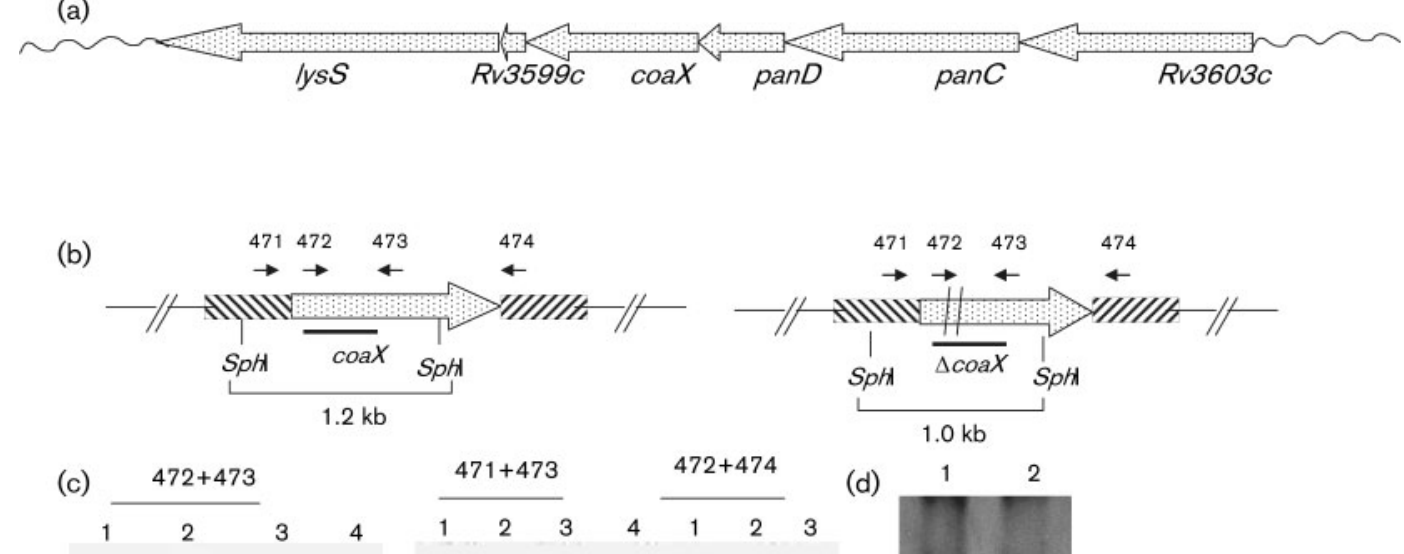

(d)
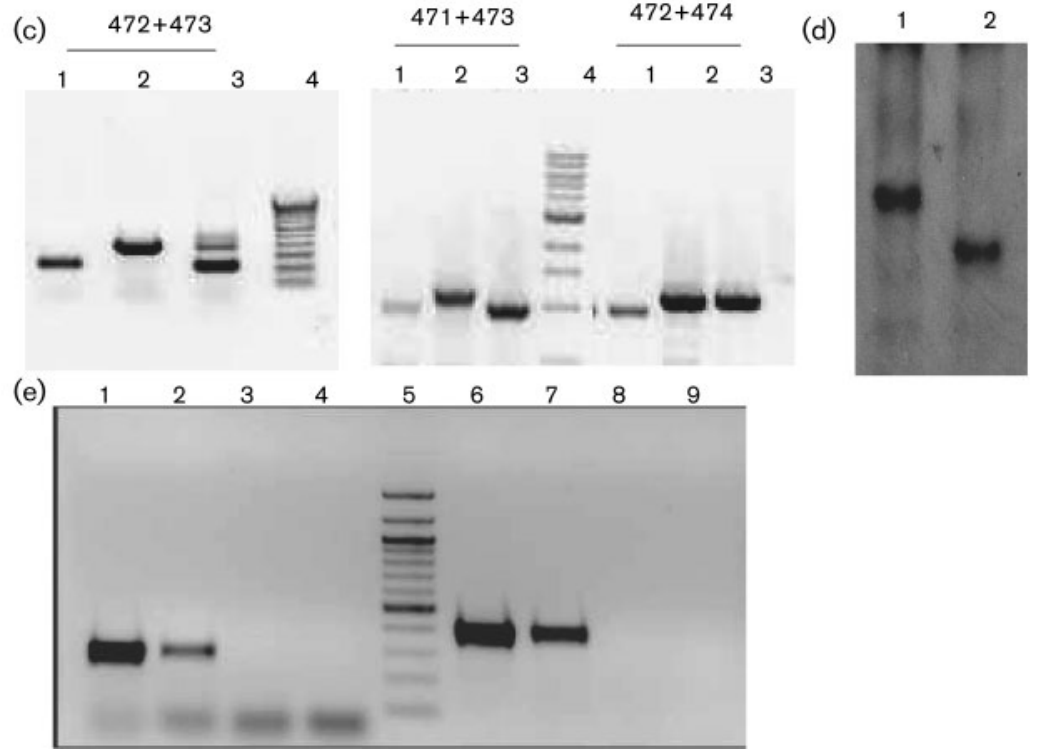

Fig. 2. Gene KO of coaX. (a) Genomic organization of coaX in the M. tuberculosis genome. (b) Comparison of the sizes of the wild-type and the mutant alleles of coaX, the binding sites of primers used for amplification of SCO and DCO recombinants by PCR and the position of restriction enzyme used for digestion of DNA for Southern blotting. The probe used for hybridization is shown as a solid line. (c) Analysis of Mtb/coaX/SCO27 and Mtb/coaX/KO by PCR. Lanes: 1, Mtb/coaX/KO; 2, H37Rv; 3, Mtb/coaX/SCO27; 4, molecular size marker. (d) Southern blot of H37Rv (lane 1) and Mtb/coaX/KO (2). The primer sequences are: 471，5'-AGCGCATGCGGCAACGGCTGG-3'; 472, 5'-AGTGGCGGATACGCACCGAAT-3'; 473, 5'-GGCATCGGAAGACACCTGCAC-3'; 474, 5'-GATCACCGCGCCATGCACGTA-3'. (e) Detection of coaA and coaX transcripts in M. tuberculosis by RT-PCR. Lanes 1-4, coaA; lanes 6-9, coaX. 1, 6, genomic DNA; 2, 7, RT template; 3, 8, RT-minus template; 4, 9, no template; 5 , molecular size marker.

and bacteria were diluted appropriately in cold, sterile PBS. The mice were infected with the wild-type, the KO mutant or the complemented M. tuberculosis strain by intravenous inoculation into the tail vein to obtain $10^{5}$ c.f.u. per animal. The animals were sacrificed at intervals of 1 week post-infection, and the bacterial counts present in the organs were determined by plating the lung and spleen homogenates on $7 \mathrm{H} 11$ agar.

\section{RESULTS}

\section{Mycobacteria carry both type I and type III pantothenate kinases}

Type III is the most common type of pantothenate kinase found in bacteria followed by type I and II (Yang et al., 2006). Several bacteria harbour combinations of type I and
III or type II and III enzymes. Mycobacteria, including $M$. tuberculosis, M. leprae, M. bovis, M. avium and M. smegmatis, show the presence of genes encoding type I and type III pantothenate kinase in their genomes. Genomic organization of coaA in M. tuberculosis revealed that it is present as a single gene, whereas coaX forms an operon with genes involved in pantothenate biosynthesis (Figs 1a and 2a). The presence of coaX in the pantothenate biosynthesis operon suggests that it plays a role in this pathway.

\section{CoaA (Rv1092c) is essential for the survival of M. tuberculosis}

Based upon the existing knowledge of in vitro gene essentiality of pantothenate kinases in various bacteria 
(Dunn \& Snell 1979; Vallari \& Rock, 1987; Wood \& Friedman, 2000; Liberati et al., 2006: Paige et al., 2008), it can be speculated that either of the two kinase coding genes (coaA or coaX) could potentially be capable of providing the essential enzymic function for the survival of $M$. tuberculosis. In order to prove whether either of them could keep the cells alive in the absence of other isoforms (as in $B$. subtilis) or only one is essential even in the presence of the other isoform (as in B. anthracis), we attempted to inactivate the coaA and coaX genes of $M$. tuberculosis individually. This was achieved by homologous recombination using a deleted copy of either of the genes using the procedure described by Parish \& Stoker (2000). The ability to obtain a gene $\mathrm{KO}$ only in the presence of an additional copy of the gene in the merodiploid strain and not in the wild-type M. tuberculosis $\mathrm{H} 37 \mathrm{Rv}$ was taken as evidence of the essentiality of the gene. The recombination substrate for coaA gene $\mathrm{KO}$ on pAZI0228 consisted of a mutant coaA gene with a 739 bp markerless deletion (7746) with $810 \mathrm{bp}$ upstream and $1078 \mathrm{bp}$ downstream flanking the deleted region. Additionally, a construct with a $h y g^{r}$ insertion in the deleted region of coaA (pAZI0222) was also tried for allele exchange. As described in Methods, the construct was designed in such a way that it would not express any intact CoaA protein. The single cross-over (SCO) recombinants obtained by transformation of $M$. tuberculosis with pAZI0228 were confirmed by PCR using primers indicated in Fig. 1(b). One of the recombinants showing integration in the proper locus $(\mathrm{Mtb} / \mathrm{coaA} / \mathrm{SCO} 3)$ was further taken up for replacement of the wild-type coaA gene by the deleted copy of the gene by a double cross-over (DCO) recombination event. Mtb/coaA/SCO3 was grown in 7H9 broth in the absence of hygromycin for 3 weeks and subsequently plated on $7 \mathrm{H} 9$ plates containing $2 \%$ sucrose. The loss of plasmid integrated in the chromosome was monitored by amplification of $h y g^{r}$ and the $s a c B$ genes by PCR. Of 200 colonies screened (150 from $h y g^{r}$ insertion and 50 from deletion construct), 140 had lost the plasmid, indicating that $70 \%$ of the colonies had undergone recombination by a DCO event. The putative DCO recombinants (showing absence of $s a c B$ and $h y g^{r}$ genes) were further screened for the presence of the mutated copy of the gene in the chromosome by PCR using primers shown in Fig. 1(b). None of the colonies showed the presence of a mutant copy of coaA in the genome. Despite a high frequency of DCO recombination events, the inability to generate a $\mathrm{KO}$ mutant indicated that the coaA gene is likely to be essential for the survival of M. tuberculosis. In order to confirm this experimentally, the coaA gene $\mathrm{KO}$ was attempted in a merodiploid background. For this purpose, pAZI0234, which carries the M. tuberculosis coaA gene under the control of hsp60 promoter in the mycobacterial integrating vector, pAZI0272, was electroporated into $\mathrm{Mtb} / \mathrm{coaA} / \mathrm{SCO} 3$ strain as described above and the transformed cells were plated on 7H9 agar containing hygromycin and kanamycin. The integration of pAZI0234 into the chromosome of the Mtb/coaA/SCO3 was confirmed by amplification of the $\mathrm{kan}^{r}$ gene (data not shown) and the strain was designated $\mathrm{Mtb} / \mathrm{coaA} / \mathrm{SCO} 3 /$ mero. As described above for $\mathrm{Mtb} / \mathrm{coaA} / \mathrm{SCO} 3, \mathrm{Mtb} / \mathrm{coaA} /$ SCO3/mero culture was grown up and plated on $7 \mathrm{H} 9$ plates for DCO recombinants, and the loss of the integrated plasmid was monitored by PCR. The frequency of DCO recombination in $\mathrm{Mtb} / \mathrm{coaA} / \mathrm{SCO} 3$ and $\mathrm{Mtb} / \mathrm{coaA} / \mathrm{SCO} 3 /$ mero was in a similar range. The colonies that had lost the plasmid were further screened for the presence of a deleted copy of coaA by PCR by using gene-specific primers (Fig. 1c). In contrast with $\mathrm{Mtb} / \mathrm{SCO} 3, \mathrm{DCO}$ recombination in $\mathrm{Mtb} / \mathrm{SCO} 3 /$ mero resulted in replacement of the wild-type coaA gene by a deleted copy in $20 \%$ of the colonies ( 18 of 88). The presence of a deleted copy of the coaA gene in the chromosome of one such DCO recombinant (Mtb/coaA/ $\mathrm{KO}$ ) was finally confirmed by Southern blotting using a 340 bp PCR product as a probe. As shown in Fig. 1(d), $\mathrm{Mtb} / \mathrm{coaA} / \mathrm{KO}$ showed the presence of a smaller DNA fragment corresponding to the deleted coaA in addition to the full-length wild-type coaA gene present in the att site.

In order to gain more confidence into the essentiality of CoaA in M. tuberculosis, the coaA genes present at the att site in $\mathrm{H} 37 \mathrm{Rv}$ and $\mathrm{Mtb} / \mathrm{coaA} / \mathrm{KO}$ were subjected to excision as described in Methods. The H37Rv cultures could be transformed with excisionase expressing pAZI0288 at high frequency $\left(5 \times 10^{3}\right.$ c.f.u. per electroporation), whereas only six colonies were obtained in the case of $\mathrm{Mtb} / \mathrm{coaA} / \mathrm{KO}$. These six colonies were found to have retained the integrated plasmid at the att site (data not shown). Both $\mathrm{H} 37 \mathrm{Rv}$ and $\mathrm{Mtb} / \mathrm{coaA} / \mathrm{KO}$ strains could be transformed with a control plasmid with equal frequency. This clearly demonstrated that at least one copy of the intact gene has to be present inside the cell for the colonies to grow in vitro. This clearly proved that coaA is essential for survival of $M$. tuberculosis, and the coaX gene present in this organism is unable to complement the loss of coaA, suggesting non-redundancy of their functions under the conditions tested.

\section{coaX (Rv3600c) gene is not essential for the survival of $M$. tuberculosis}

CoaX of $M$. tuberculosis has been predicted to be a pantothenate kinase based upon its high degree of amino acid homology to the biochemically characterized type III isoform of pantothenate kinase of $H$. pylori (Brand \& Strauss, 2005). The coaX gene has been shown to be essential for survival of $B$. anthracis, $P$. aeruginosa and Bordetella pertussis (Wood \& Friedman, 2000; Liberati et al., 2006; Paige et al., 2008). In order to assess whether CoaX is essential for the survival of $M$. tuberculosis, we decided to inactivate the coaX gene by homologous recombination using the strategy applied for coaA $\mathrm{KO}$ described above. The suicide plasmid pAZI0294 carried a mutant copy of coaX, which could only code for $79 \mathrm{~N}$ terminal amino acids (out of 272) as a result of a deletion of $181 \mathrm{bp}$ after bp 237, which renders the downstream sequence out of frame. The SCO recombinants obtained 
by transformation of pAZI0294 into $M$. tuberculosis were confirmed by PCR (Fig. 2c) and one of them (Mtb/coaX/ SCO27) was grown in $7 \mathrm{H} 9$ and plated on $2 \%$ sucrose to obtain DCOs. Of the 130 sucrose-resistant resistant colonies selected for analysis, more than $90 \%$ were found to have lost the plasmid, as determined by the absence of $s a c B$ and $h y g^{r}$ genes in PCR analysis of DCO colonies (data not shown). Analysis of these colonies by gene-specific PCRs using primers 472 and 473 showed that 4 of 130 had only the mutant copy of the gene while the remainder had retained the wild-type copy of the gene. Further confirmation of the absence of wild-type coaX gene copy in one of the mutants $(\mathrm{Mtb} / \mathrm{coaX} / \mathrm{KO})$ was done by PCR using primers shown in Fig. 2(c). The absence of wild-type coaX gene in $\mathrm{Mtb} / \mathrm{coax} / \mathrm{KO}$ was also confirmed by Southern blot analysis using a PCR fragment corresponding to bp 85-486 of the coaX gene as a probe. As shown in Fig. 2(d), the size of the SphI-XhoI fragment was found to be smaller than the fragment in the wild- type $M$. tuberculosis H37Rv. Thus it was clearly demonstrated that in contrast with coaA, the coaX gene could be deleted in the wild-type $M$. tuberculosis, proving that it is nonessential for survival. Mtb/coaX/KO was further studied for its phenotype under in vitro, ex vivo and in vivo growth conditions.

Is it possible that coaX happens to be non-essential in $M$. tuberculosis because it is not expressed? In order to answer this question, RT-PCR analysis of mRNA isolated from actively growing $M$. tuberculosis cells was performed. Amplification of both coaA and coaX cDNAs gave rise to DNA fragments of the expected sizes (292 bp and $401 \mathrm{bp}$, respectively; Fig. 2e). No DNA fragments were generated in
RT-minus or template minus samples (Fig. 2e, lanes 3, 4, 8 and 9) confirming the amplification of specific transcripts. This shows that both coaA and the coaX genes are transcribed in $M$. tuberculosis, and the non-essential nature of coaX cannot be ascribed to the lack of expression.

\section{CoaX is not required for survival in macrophages and mice}

$\mathrm{Mtb} / \mathrm{coaX} / \mathrm{KO}$ did not show any significant growth defect in 7H9 medium (data not shown). Next, we asked whether CoaX could be critical for survival under ex vivo and/or in vivo conditions. In order to answer this question, we first compared the growth and survival of the wild-type and the coaX mutant in BMDMs. The infected macrophages were lysed and plated to estimate the bacterial load on days 0,3 , 7 and 10. There was no difference in the growth characteristics of wild-type and $\mathrm{Mtb} / \mathrm{coaX} / \mathrm{KO}$ (Fig. 3a), as in both cases the bacterial number remained unchanged between days 0 and 10. In order to understand the contribution of CoaX towards survival in vivo, growth kinetics of the $\mathrm{Mtb} / \mathrm{coaX} / \mathrm{KO}$ mutant were compared with the wild-type $M$. tuberculosis. The c.f.u. values obtained from lung and spleen homogenates of mice infected with wild-type, mutant or the complemented strains were similar (Fig. $3 \mathrm{~b}$ and c). Statistical analysis (Student's $t$-test) of the c.f.u. values obtained from lungs of wild-type and the mutant on day 56 showed that these values were significantly different $(P=0.007)$, though the difference in the c.f.u. values of similar samples from spleens was not significant $(P>0.5)$. The course of infection of the complemented strain in the lungs and the spleens was similar to that of the wild-type strain, though the starting
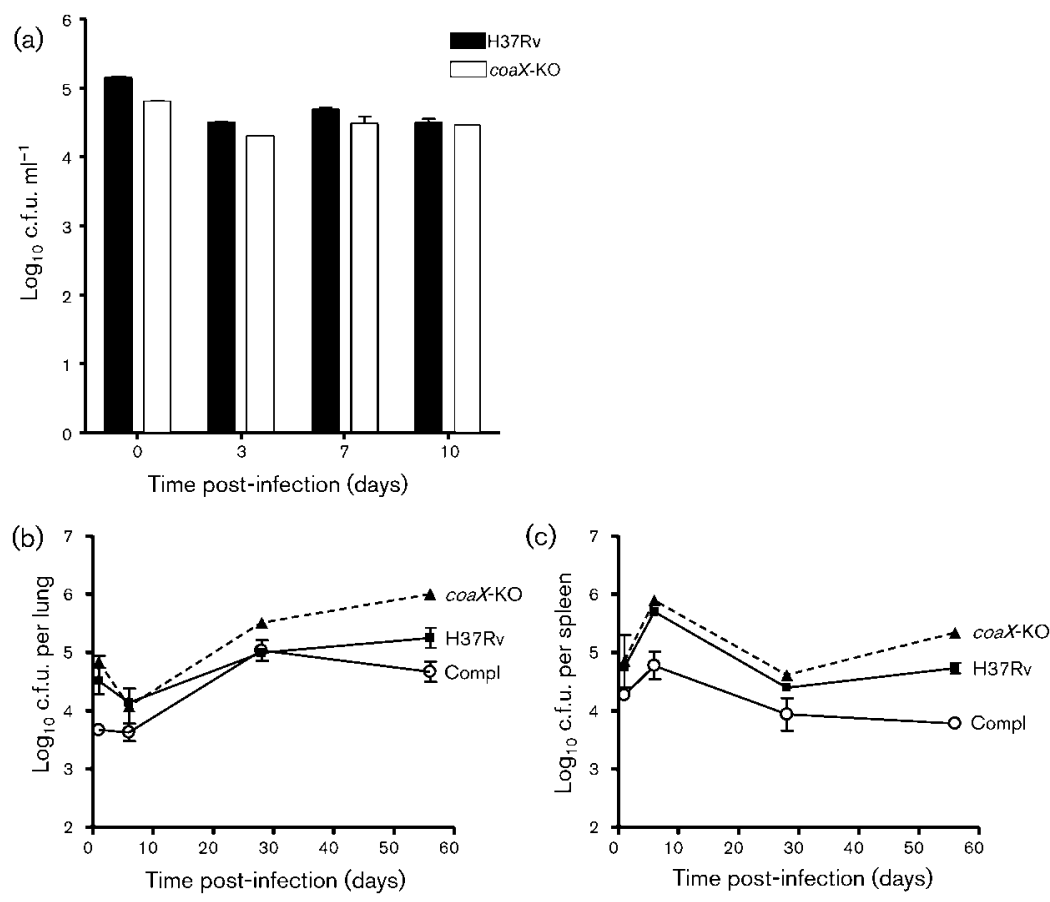

Fig. 3. Ex vivo and in vivo phenotypes of coaX KO. (a) M. tuberculosis H37Rv and coaX KO obtained from infected BMDM. (b) and (c) $\mathrm{H} 37 \mathrm{Rv}, \mathrm{Mtb} / \mathrm{coaX} / \mathrm{KO}$ and Mtb/coaX/KO/ complemented strains obtained from lungs and spleen of infected mice. 
c.f.u. of the complemented strain was slightly less than the wild-type. Small variations in initial bacterial loads were seen earlier and are not expected to influence the course of infection (Sun et al., 2004). Overall, the absence of coaX does not lead to any kind of change in virulence in $M$. tuberculosis. Thus, it was quite puzzling to see that CoaX, which is non-essential for survival in vitro, does not contribute towards survival of $M$. tuberculosis under ex vivo or in vivo conditions.

\section{CoaA of M. tuberculosis complements a CoaA ts mutant of $E$. coli while CoaX does not}

In B. subtilis, both CoaX and CoaA were shown to be functional by their ability to keep the cells alive in the absence of the other isoforms, indicating that both type I and type III pantothenate kinases are active in B. subtilis. Additionally, both coaA and coaX could rescue the growth of a CoaA ts mutant of $E$. coli at non-permissive temperature (Yocum \& Patterson, 2004). Since we found that $\mathrm{CoaX}$ was non-essential for survival in vitro or in vivo and could not complement the loss of CoaA in $M$. tuberculosis, we wanted to see whether this protein could function as a pantothenate kinase in E. coli. For this purpose we tested the ability of $M$. tuberculosis CoaA and CoaX to complement a ts mutation in CoaA of E. coli at non-permissive temperature. The individual plasmids coding for $M$. tuberculosis CoaX and CoaA under the control of an arabinose-inducible promoter (pAZI0371 and pAZI0372) were transformed into E. coli DV62 at $30{ }^{\circ} \mathrm{C}$ and plated at 30 and $42{ }^{\circ} \mathrm{C}$ in the presence of arabinose. It was found that $E$. coli DV62 transformed with any of the plasmids was able to grow at $30{ }^{\circ} \mathrm{C}$ whereas only M. tuberculosis CoaA was able to support the growth of E. coli DV62 at $42{ }^{\circ} \mathrm{C}$ (Fig. 4a). The ability of M. tuberculosis CoaA to complement a ts CoaA of E. coli is consistent with the earlier findings of Kumar et al. (2007). In order to rule out the possibility that the lack of protein expression or lack of

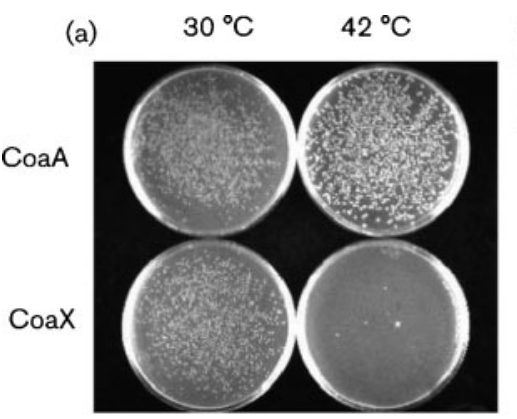

(b) 1 2

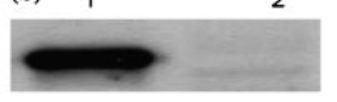

Fig. 4. Rescue of ts phenotype of $E$. coli DV62 by CoaA and CoaX. (a) Growth of E. coli DV62 transformed with plasmids expressing $M$. tuberculosis CoaA and CoaX grown at 30 and $42{ }^{\circ} \mathrm{C}$. (b) Western blots showing the expression of CoaX in E. coli DV62 at $30{ }^{\circ} \mathrm{C}$. Lanes: 1 , CoaX; 2 , vector control. soluble protein is responsible for the inability of the coaX gene to complement the ts phenotype in E. coli, we analysed the protein expression in E. coli DV62 pAZI0371 by Western blotting using anti-CoaX antibodies. As shown in Fig. 4(b), sufficient amounts of CoaX protein were present in the cytosolic fractions of cell lysates. This set of experiments proved that CoaX of $M$. tuberculosis does not function as a pantothenate kinase in E. coli.

\section{CoaX of M. tuberculosis lacks pantothenate kinase activity in vitro}

In order to determine why CoaX is unable to function in M. tuberculosis or in E. coli, we analysed the recombinant CoaX for pantothenate kinase activity. CoaX purified from E. coli was assayed for enzymic activity by the PK-LDH coupled assay described in Methods. No enzymic activity could be observed under standard assay conditions using $300 \mathrm{nM}$ of the enzyme. On the other hand, CoaA showed the expected enzymic activity under these assay conditions (Fig. 5b). Analysis of CoaA kinetic parameters revealed that its catalytic constant (kcat) value $\left(3.17 \mathrm{~s}^{-1}\right)$ was in a similar range to those reported for type III pantothenate kinases of $H$. pylori and B. subtilis and the type II kinase of S. aureus (Brand \& Strauss, 2005). Since it had been observed previously that type III pantothenate kinase had higher $K_{\mathrm{m}}$ values for ATP (Brand \& Strauss, 2005), the assays were repeated with increasing amounts of ATP (up to $12.5 \mathrm{mM})$ and pantothenate $(0-300 \mu \mathrm{M})$. However, no activity was observed under these conditions either. Increasing the concentration of CoaX (from $100 \mathrm{nM}$ to $3.2 \mu \mathrm{M}), \mathrm{MgCl}_{2}(62.5 \mathrm{mM}), \mathrm{NADH}(0.75 \mathrm{mM})$ or inclusion of potassium glutamate and ammonium chloride did not make any difference (data not shown). This suggested

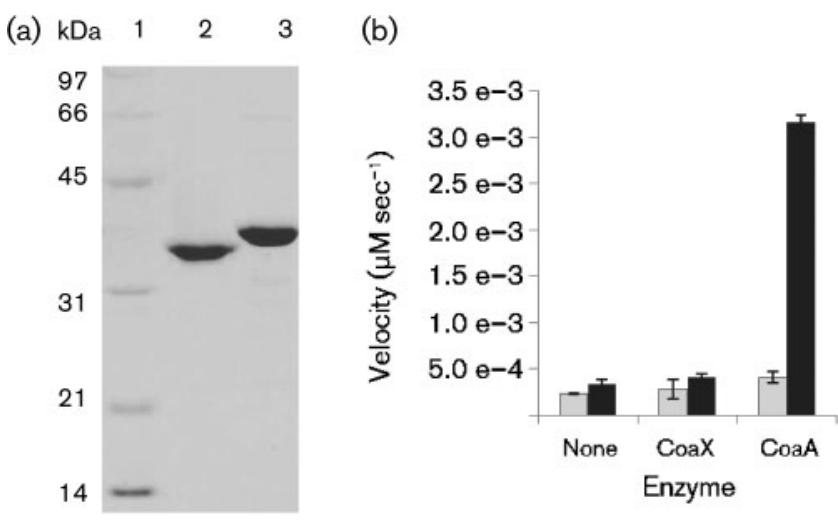

Fig. 5. Enzymic activity of CoaA and CoaX of M. tuberculosis. The purified CoaA and CoaX proteins were assayed for activity as described in Methods. (a) Purified CoaX and CoaA of M. tuberculosis. Lanes: 1, molecular size marker; 2, CoaX; 3, CoaA. (b) Pantothenate kinase activity of CoaA and CoaX with $12.5 \mathrm{mM}$ (black bars) or no (grey bars) ATP. The concentration of CoaA and CoaX used in this assay was $100 \mathrm{nM}$ and $300 \mathrm{nM}$, respectively. 
that CoaX of M. tuberculosis lacked any observable pantothenate kinase activity in vitro, and this is also consistent with the observation that CoaX of M. tuberculosis is unable to function as a pantothenate kinase in $E$. coli. Interestingly, in an earlier study, the type II pantothenate kinase of $B$. anthracis was found to have no pantothenate kinase activity and was unable to rescue the growth of the ts CoaA mutant of E. coli at a non-permissive temperature (Nicely et al., 2007).

\section{DISCUSSION}

The availability of the genome sequence of $M$. tuberculosis has thrown up enormous possibilities of finding novel drug targets. Amongst these targets, some would be essential for survival under most growth conditions (e.g. RNA polymerase), while others could only be required under certain physiological conditions (e.g. virulence factors). Inhibition of targets, which are required only for growth in vivo, is not expected to lead to growth inhibition in vitro and thus the inhibitors will have to be tested in vivo only. Also, the inhibition of targets that are not essential in vivo is not expected to result in elimination of the organism from the host. Therefore, the knowledge of in vitro and in vivo essentiality of the targets is crucial in designing compound progression strategies in drug discovery.

In organisms that possess a single isoform of pantothenate kinase, the gene encoding the lone form of pantothenate kinase is expected to be essential. This has been proven in a number of bacteria such as E. coli, $P$. aeruginosa and Bordetella pertussis (Dunn \& Snell 1979; Vallari \& Rock, 1987; Wood \& Friedman, 2000). Since all three forms of pantothenate kinase have been shown to be functionally active, it can be speculated that in organisms possessing multiple forms of pantothenate kinases, any one of the three isoforms should be sufficient for in vitro survival of the bacteria. This has been found to be true in B. subtilis, wherein it has been demonstrated that the presence of either CoaA or CoaX permits the cell to be viable (Yocum \& Patterson, 2004). However, in a related bacterium, $B$. anthracis, which possesses type II and III isoforms of pantothenate kinase (Yang et al. 2006), the coaX gene was found to be essential even in the presence of type II isoform of pantothenate kinase (Paige et al., 2008), which led the authors to propose CoaX as a drug target for developing inhibitors (Paige et al., 2008). Whether the type II pantothenate kinase of $B$. anthracis is also essential or not is not clear at this moment.

Though in vitro studies with recombinant $M$. tuberculosis CoaA have indicated that pantothenol can act as a substrate for this enzyme, thereby blocking the subsequent steps of CoA biosynthesis (Kumar et al., 2007), the in vivo effect of these analogues has not been studied. Our gene inactivation studies demonstrate that under in vitro growth conditions, CoaA is the functional pantothenate kinase in M. tuberculosis, and CoaX is not able to complement the loss of CoaA. This finding is in contrast with the situation that exists in B. subtilis. Interestingly, M. leprae, which has lost most of the non-essential genes from its genome (Cole et al., 2001), has retained both coaA and coaX, suggesting that both the isoforms might play important roles under varied physiological conditions. Since CoaX of $M$. tuberculosis is not essential in vitro, we had hypothesized that it might be required for survival in macrophages or in vivo in animals. However, the absence of any growth defect in macrophages or in mice rules out the possibility of CoaX playing a major role in survival in this animal model. The exact reason for this result is not clear at this moment. However, it is possible that these enzymes might have evolved to confer a survival advantage in human hosts and hence might not be crucial for survival in other species. Testing of the phenotype of the coaX mutant in other animal species might help to answer this question. Differences in phenotypes of $M$. tuberculosis mutants in various animal species have been reported previously (Converse et al., 2009).

The inability of CoaX to complement a CoaA ts mutant of E. coli and the lack of any demonstrable biochemical activity in vitro raises the question of whether CoaX possesses pantothenate kinase activity or not. It might also be possible that it requires an environment for activity that is not present in vitro or in heterologous systems. Comparison of the amino acid sequence of $M$. tuberculosis CoaX with CoaX of $H$. pylori and B. anthracis, in which pantothenate kinase activity has been demonstrated (Yang et al., 2006), show that the substrate-binding residues and other characteristic motifs are conserved amongst the enzymes from these species. Thus, lack of functional and biochemical activity in CoaX of M. tuberculosis cannot be explained on the basis of the amino acid sequence of the protein. Also, the existence of an entirely different enzymic activity in CoaX of $M$. tuberculosis cannot be ruled out. The presence of a seemingly inactive pantothenate kinase in two intracellular pathogens, $B$. anthracis (Nicely et al., 2007) and M. tuberculosis, both carrying two isoforms of this enzyme, is intriguing and lacks any valid explanation at this moment. Further biochemical and structural studies will be required to find an answer to this complex question.

The presence of multiple isoforms of enzymes is quite common in M. tuberculosis, which is perhaps reflected in the bigger genome size of this organism compared with other pathogenic bacteria (Cole et al., 1998). Glutamine synthases, DNA ligases, ketoacyl synthases, thymidylate synthase and methionine aminopeptidases are some of the examples of enzymes that exist as multiple isoforms in $M$. tuberculosis. The redundancy of putative drug targets in $M$. tuberculosis calls for stringent target validation efforts before commencement of target-based drug discovery programs to identify potential inhibitors. In summary, we have established that CoaA is a valid drug target in $M$. tuberculosis, whereas inhibition of CoaX will not result in growth inhibition in vitro or in vivo. This, along with the 
recently solved crystal structure of $M$. tuberculosis CoaA protein (Das et al., 2006), will be of great value in identification and characterization of CoaA inhibitors.

\section{ACKNOWLEDGEMENTS}

We thank Tanya Parish (Queen Mary's School of Medicine and Dentistry, London) for providing pGOAL19 and pJL28 and J. Beckwith (Harvard Medical School, Boston MA) for pBAD24. Thanks are due to Madhavapeddi Prashanti for reviewing the manuscript and Sheshagiri Gaonkar for help in statistical analysis. We thank Shreeharsha for subcloning the KO constructs, Devang Patel for cloning the coaA and coaX genes, Debasmita Sarkar for CoaA purification and Sowmya Bharath for help with animal studies. We appreciate the support and encouragement provided by T. S. Balganesh and Kaveri Das.

\section{REFERENCES}

Awasthy, D., Gaonkar, S., Shandil, R. K., Yadav, R., Bharath, S., Marcel, N., Subbulakshmi, V. \& Sharma, U. (2009). Inactivation of the ilvB1 gene in Mycobacterium tuberculosis leads to branched-chain amino acid auxotrophy and attenuation of virulence in mice. Microbiology 155, 2978-2987.

Brand, L. A. \& Strauss, E. (2005). Characterization of a new pantothenate kinase isoform from Helicobacter pylori. J Biol Chem 280, 20185-20188.

Chan, E. D. \& Iseman, M. D. (2008). Multidrug-resistant and extensively drug-resistant tuberculosis: a review. Curr Opin Infect Dis 21, 587-595.

Choudhry, A. E., Mandichak, T. L., Broskey, J. P., Egolf, R. W., Kinsland, C., Begley, T. P., Seefeld, M. A., Ku, T. W., Brown, J. R. \& other authors (2003). Inhibitors of pantothenate kinase: novel antibiotics for staphylococcal infections. Antimicrob Agents Chemother 47, 2051-2055.

Cole, S. T., Brosch, R., Parkhill, J., Garnier, T., Churcher, C., Harris, D., Gordon, S. V., Eiglmeier, K., Gas, S. \& other authors (1998). Deciphering the biology of Mycobacterium tuberculosis from the complete genome sequence. Nature 393, 537-544.

Cole, S. T., Eiglmeier, K., Parkhill, J., James, K. D., Thomson, N. R., Wheeler, P. R., Honoré, N., Garnier, T., Churcher, C. \& other authors (2001). Massive gene decay in the leprosy bacillus. Nature 409, 1007-1011.

Converse, P. J., Karakousis, P. C., Klinkenberg, L. G., Kesavan, A. K., Ly, L. H., Allen, S. S., Grosset, J. H., Jain, S. K., Lamichhane, G. \& other authors (2009). Role of the dosR-dosS two-component regulatory system in Mycobacterium tuberculosis virulence in three animal models. Infect Immun 77, 1230-1237.

Das, S., Kumar, P., Bhor, V., Surolia, A. \& Vijayan, M. (2006). Invariance and variability in bacterial PanK: a study based on the crystal structure of Mycobacterium tuberculosis PanK. Acta Crystallogr D Biol Crystallogr 62, 628-638.

Dunn, S. D. \& Snell, E. E. (1979). Isolation of temperature-sensitive pantothenate kinase mutants of Salmonella typhimurium and mapping of the coaA gene. J Bacteriol 140, 805-808.

Gerdes, S. Y., Scholle, M. D., D'Souza, M., Bernal, A., Baev, M. V., Farrell, M., Kurnasov, O. V., Daugherty, M. D., Mseeh, F. \& other authors (2002). From genetic footprinting to antimicrobial drug targets: examples in cofactor biosynthetic pathways. J Bacteriol 184, 4555-4572.

Guzman, L. M., Belin, D., Carson, M. J. \& Beckwith, J. (1995). Tight regulation, modulation, and high-level expression by vectors containing the arabinose PBAD promoter. J Bacteriol 177, 4121-4130.
Hoft, D. F. (2008). Tuberculosis vaccine development: goals, immunological design, and evaluation. Lancet 372, 164-175.

Jackowski, S. \& Rock, C. O. (1981). Regulation of coenzyme A biosynthesis. J Bacteriol 148, 926-932.

Kumar, P., Chhibber, M. \& Surolia, A. (2007). How pantothenol intervenes in Coenzyme-A biosynthesis of Mycobacterium tuberculosis. Biochem Biophys Res Commun 361, 903-909.

Leonardi, R., Chohnan, S., Zhang, Y. M., Virga, K. G., Lee, R. E., Rock, C. O. \& Jackowski, S. (2005). A pantothenate kinase from Staphylococcus aureus refractory to feedback regulation by coenzyme A. J Biol Chem 280, 3314-3322.

Liberati, N. T., Urbach, J. M., Miyata, S., Lee, D. G., Drenkard, E., Wu, G., Villanueva, J., Wei, T. \& Ausubel, F. M. (2006). An ordered, nonredundant library of Pseudomonas aeruginosa strain PA14 transposon insertion mutants. Proc Natl Acad Sci U S A 103, 2833-2838.

Muñoz-Elías, E. J. \& McKinney, J. D. (2005). Mycobacterium tuberculosis isocitrate lyases 1 and 2 are jointly required for in vivo growth and virulence. Nat Med 11, 638-644.

Nicely, N. I., Parsonage, D., Paige, C., Newton, G. L., Fahey, R. C., Leonardi, R., Jackowski, S., Mallett, T. C. \& Claiborne, A. (2007). Structure of the type III pantothenate kinase from Bacillus anthracis at 2.0 A resolution: implications for coenzyme A-dependent redox biology. Biochemistry 46, 3234-3245.

Paige, C., Reid, S. D., Hanna, P. C. \& Claiborne, A. (2008). The type III pantothenate kinase encoded by coaX is essential for growth of Bacillus anthracis. J Bacteriol 190, 6271-6275.

Parish, T. \& Stoker, N. G. (2000). Use of a flexible cassette method to generate a double unmarked Mycobacterium tuberculosis tlyA plcABC mutant by gene replacement. Microbiology 146, 1969-1975.

Parish, T., Lewis, J. \& Stoker, N. G. (2001). Use of the mycobacteriophage L5 excisionase in Mycobacterium tuberculosis to demonstrate gene essentiality. Tuberculosis (Edinb) 81, 359-364.

Rivers, E. C. \& Mancera, R. L. (2008). New anti-tuberculosis drugs in clinical trials with novel mechanisms of action. Drug Discov Today 13, 1090-1098.

Rock, C. O., Park, H. W. \& Jackowski, S. (2003). Role of feedback regulation of pantothenate kinase (CoaA) in control of coenzyme A levels in Escherichia coli. J Bacteriol 185, 3410-3415.

Saliba, K. J., Ferru, I. \& Kirk, K. (2005). Provitamin B5 (pantothenol) inhibits growth of the intraerythrocytic malaria parasite. Antimicrob Agents Chemother 49, 632-637.

Snell, E. E. \& Shive, W. (1945). Growth inhibition by analogues of pantothenic acid. Pantothenyl alcohol and related compounds. J Biol Chem 158, 551-559.

Spry, C., Chai, C. L., Kirk, K. \& Saliba, K. J. (2005). A class of pantothenic acid analogs inhibits Plasmodium falciparum pantothenate kinase and represses the proliferation of malaria parasites. Antimicrob Agents Chemother 49, 4649-4657.

Spry, C., Kirk, K. \& Saliba, K. J. (2008). Coenzyme A biosynthesis: an antimicrobial drug target. FEMS Microbiol Rev 32, 56-106.

Sun, R., Converse, P. J., Ko, C., Tyagi, S., Morrison, N. E. \& Bishai, W. R. (2004). Mycobacterium tuberculosis ECF sigma factor sigC is required for lethality in mice and for the conditional expression of a defined gene set. Mol Microbiol 52, 25-38.

Vallari, D. S. \& Rock, C. O. (1987). Isolation and characterization of temperature-sensitive pantothenate kinase (coaA) mutants of Escherichia coli. J Bacteriol 169, 5795-5800.

Wards, B. J. \& Collins, D. M. (1996). Electroporation at elevated temperatures substantially improves transformation efficiency of slow-growing mycobacteria. FEMS Microbiol Lett 145, 101-105. 
Wood, G. E. \& Friedman, R. L. (2000). The Bvg accessory factor (Baf) enhances pertussis toxin expression in Escherichia coli and is essential for Bordetella pertussis viability. FEMS Microbiol Lett 193, 25-30.

Yang, K., Eyobo, Y., Brand, L. A., Martynowski, D., Tomchick, D., Strauss, E. \& Zhang, H. (2006). Crystal structure of a type III pantothenate kinase: insight into the mechanism of an essential coenzyme A biosynthetic enzyme universally distributed in bacteria. J Bacteriol 188, 5532-5540.

Yang, K., Strauss, E., Huerta, C. \& Zhang, H. (2008). Structural basis for substrate binding and the catalytic mechanism of type III pantothenate kinase. Biochemistry 47, 1369-1380.
Yocum, R. R. \& Patterson, T. A. (2004). US Patent 6,830,898.

Zhang, Y. M., Frank, M. W., Virga, K. G., Lee, R. E., Rock, C. O. \& Jackowski, S. (2004). Acyl carrier protein is a cellular target for the antibacterial action of the pantothenamide class of pantothenate antimetabolites. J Biol Chem 279, 50969-50975.

Zhao, L., Allanson, N. M., Thomson, S. P., Maclean, J. K., Barker, J. J., Primrose, W. U., Tyler, P. D. \& Lewendon, A. (2003). Inhibitors of phosphopantetheine adenylyltransferase. Eur J Med Chem 38, 345349.

Edited by: G. R. Stewart 